\title{
Reduced Amplitude of Low-Frequency Brain Oscillations in the Psychosis Risk Syndrome and Early Illness Schizophrenia
}

\author{
Susanna L Fryer ${ }^{1,2}$, Brian J Roach², Katherine Wiley², Rachel L Loewy', Judy M Ford ${ }^{1,2}$ and \\ Daniel H Mathalon*, I,2 \\ 'Department of Psychiatry, University of California, San Francisco, San Francisco, CA, USA; ${ }^{2}$ San Francisco VA Medical Center, San Francisco, \\ CA, USA
}

\begin{abstract}
Low-frequency oscillations (LFOs) of the blood oxygen level-dependent (BOLD) signal are gaining interest as potential biomarkers sensitive to neuropsychiatric pathology. Schizophrenia has been associated with alterations in intrinsic LFOs that covary with cognitive deficits and symptoms. However, the extent to which LFO dysfunction is present before schizophrenia illness onset remains unknown. Resting-state FMRI data were collected from clinical high-risk $(C H R ; n=45)$ youth, early illness schizophrenia $(E S Z ; n=74)$ patients, and healthy controls (HCs; $n=85$ ) aged 12-35 years. Age-adjusted voxelwise fractional amplitude of low-frequency fluctuations (fALFF; $0.01-0.08 \mathrm{~Hz}$ ) of the BOLD signal was compared among the three groups. Main effects of Group $(p<0.005$ height threshold, familywise error cluster-level corrected $p<0.05)$ were followed up via Tukey-corrected pairwise comparisons. Significant main effects of Group $(p<0.05)$ revealed decreased fALFF in ESZ and CHR groups relative to HCs, with values in the CHR group falling between those of ESZ and $\mathrm{HC}$ groups. These differences were identified primarily in posterior cortex, including temporoparietal regions, extending into occipital and cerebellar lobes. Less LFO activity was related to greater symptom severity in both CHR and ESZ groups in several of these posterior cortical regions. These data support an intermediate phenotype of reduced posterior cortical LFO amplitude in CHR individuals, with resting fALFF values smaller than in HCs but higher than in ESZ patients. Findings indicate that LFO magnitude alterations relate to clinical symptoms and predate psychosis onset but are more pronounced in the early stages of schizophrenia.

Neuropsychopharmacology (2016) 4I, 2388-2398; doi:I0.1038/npp.20 I6.5 I; published online II May 20I6
\end{abstract}

\section{INTRODUCTION}

Dysconnectivity has been proposed as a critical pathophysiological mechanism in schizophrenia (Andreasen, 1999; Cannon, 2015; Friston, 1998). Functional connectivity studies in schizophrenia generally support dysconnectionist models by identifying abnormal regional connectivity patterns across task and resting conditions, often in association with symptoms and cognitive deficits (see Narr and Leaver, 2015). Functional magnetic resonance imaging (FMRI)-based connectivity measures reflect temporal synchronization of low-frequency oscillations (LFOs) of the blood oxygen level-dependent (BOLD) signal between brain regions (Biswal et al, 1995). Unlike interregional functional connectivity measures, the amplitude of low-frequency fluctuation (ALFF) indexes the intraregional magnitude of BOLD oscillations (Zang et al, 2007). These infraslow oscillations $(\approx 0.01$ to $.1 \mathrm{~Hz})$ fall within the slowest activities of a taxonomy of brain oscillations whose powerlaw distributed frequency structure is highly conserved

* Correspondence: Dr DH Mathalon, San Francisco VA Medical Centerl Psychiatry Service (II6D), 4150 Clement Street, San Francisco, CA 94I2I, USA, Tel: +I 415 22I 48I0, x23860, Fax: + 415750 6622, E-mail: daniel.mathalon@ucsf.edu

Received 27 January 2016; revised I 8 March 2016; accepted 25 March 2016; accepted article preview online 12 April 2016 across mammalian species and includes faster activities more commonly studied with electrophysiological methods (Buzsáki et al, 2013). Neuro-oscillatory rhythms are thought to have roles that include biasing input selection, neuronal assembly formation, and synaptic plasticity, implying an exquisite dependence on temporal synchronization of these fundamental neural processes (Buzsáki et al, 2013; He et al, 2008). Accordingly, there is increasing focus in neuroscience on investigating the temporal organization of brain activity, and understanding neural synchrony alterations as potential pathophysiological mechanisms in neuropsychiatric disorders (Buzsáki et al, 2013; Mathalon and Sohal, 2015), including schizophrenia (Roach and Mathalon, 2008; Uhlhaas et al, 2008).

Though hemodynamically generated, BOLD LFOs relate to underlying electrophysiology of slow changes in cortical potentials. In humans, BOLD LFOs in sensorimotor regions exhibit similar spatial correlation structure to intracranial electrocorticographic recordings of slow $(1-4 \mathrm{~Hz}$ and $<0.5 \mathrm{~Hz})$ cortical potentials as well as slow $(<0.1 \mathrm{~Hz})$ fluctuations of gamma power (He et al, 2008). Non-human primate local field potential recordings have shown infraslow fluctuations of band-limited power over seconds to minutes $(<0.1 \mathrm{~Hz})$, particularly in the gamma band, to have high spatial coherence across distal regions (Leopold et al, 2003), consistent with the temporal and spatial characteristics of 
BOLD LFOs. Resting LFOs correspond with similarly scaled fluctuations in behavioral output, suggesting a relationship between fluctuations of intrinsic brain activity and performance variability (Fox et al, 2007; Palva and Palva, 2012). Though the neurophysiological origin of LFOs is unclear, these slow spontaneous neuro-oscillatory rhythms likely reflect anatomically widespread fluctuations in cortical excitability, whereas faster neuro-oscillations reflect more local changes in neuronal excitability ( $\mathrm{He}$ et al, 2008). Because the phase of slow oscillations modulates the magnitude of faster oscillatory classes (ie, cross-frequency coupling), there is a potential modulatory role for LFOs in the organization of faster activities (Buzsáki et al, 2013). Spontaneous infraslow $(0.01-0.1 \mathrm{~Hz})$ scalp potentials recorded at rest with electroencephalography (EEG) fall specifically within, and directly correlate with, BOLD LFOs in FMRI-defined intrinsic functional connectivity networks, suggesting that these slow neuro-oscillations may maintain or gate functional brain connectivity (Hiltunen et al, 2014).

Measures of resting BOLD LFO magnitude are gaining interest as biomarkers of schizophrenia ( $\mathrm{He}$ et al, 2013; Hoptman et al, 2010; Huang et al, 2010; Lui et al, 2010, 2015; Meda et al, 2015; Ren et al, 2013; Sui et al, 2015; Turner et al, 2013; Xu et al, 2015; Yu et al, 2013, 2014). One of the most consistent findings is decreased LFO magnitude in patients with schizophrenia, relative to healthy controls, in posterior midline and occipital regions such as the posterior cingulate, precuneus, and cuneus (Hoptman et al, 2010; Meda et al, 2015; Turner et al, 2013; Yu et al, 2014). These findings have been corroborated by a meta-analysis that reported decreases in LFO magnitude in schizophrenia in sensorimotor, posterior parietal, and occipital cortices, as well as increases in LFO magnitude in bilateral striatum, medial temporal, and medial prefrontal cortices (Xu et al, 2015). LFO alterations in schizophrenia have been associated with cognitive impairment (Fryer et al, 2015; He et al, 2013; Lui et al, 2015; Sui et al, 2015), symptom severity (Lui et al, 2015; Sui et al, 2015), and antipsychotic treatment response (Lui et al, 2010).

The neurodevelopmental hypothesis of schizophrenia (see Weinberger, 1987) supports predictions that LFO alterations will predate illness onset in adolescents and young adults at clinical high risk (CHR) for psychosis. Most studies of LFOs in schizophrenia have focused on chronic patients (Fryer et al, 2015; Hoptman et al, 2010; Lui et al, 2010, 2015; Meda et al, 2015; Sui et al, 2015; Turner et al, 2013; Yu et al, 2013, 2014), although LFO alterations have been described in antipsychotic medication-naive first-episode patients (He et al, 2013; Huang et al, 2010; Lui et al, 2010; Ren et al, 2013), suggesting that observed alterations are not a function of illness chronicity or antipsychotic medication exposure. However, the extent to which resting LFO alterations are present before psychosis onset in individuals at CHR for developing schizophrenia is currently not known. Accordingly, we examined the regional amplitudes of resting BOLD LFOs in individuals at CHR for psychosis relative to both schizophrenia patients early in their illness course (ESZ) and healthy controls (HCs). Specifically, we hypothesized that, relative to HCs, CHR individuals would show a pattern of abnormal LFO activity intermediate to alterations seen in patients with ESZ.

\section{MATERIALS AND METHODS}

\section{Participants}

CHR participants $(n=45)$ were recruited from University of California, San Francisco's (UCSF) Prodromal Assessment, Research, and Treatment Clinic. Current criteria for prospective identification of the prodromal syndrome have demonstrated inter-rater reliability and predictive validity (Miller et al, 2002, 2003; Woods et al, 2009), with conversion to a psychotic disorder occurring in $\approx 35 \%$ of CHR patients after 2-3 years of follow-up (Cannon et al, 2008). CHR patients met Criteria of Prodromal Syndromes (COPS) based on the Structured Interview for Prodromal Syndromes (SIPS) (Miller et al, 2002, 2003). COPS criteria comprise three nonmutually exclusive subsyndromes: (1) attenuated psychotic symptoms $(n=43 / 45)$, (2) brief intermittent psychotic states $(n=0 / 45)$, and (3) genetic risk with deterioration in social/ occupational functioning $(n=5 / 45)$. Current symptom severity in CHR patients was assessed using the Scale of Prodromal Symptoms (SOPS), an embedded scale within the SIPS.

ESZ patients $(n=74)$ within 5 years of illness onset (mean $=1.72 \pm 1.35$ years) were recruited from the Early Psychosis Clinic at UCSF and the community. Diagnosis of schizophrenia or schizoaffective disorder was confirmed in ESZ patients using the Structured Clinical Interview for DSM-IV (SCID) (First et al, 2002). Current symptom severity was assessed with the Positive and Negative Syndrome Scale (PANSS) (Kay et al, 1987). The majority of ESZ patients were prescribed antipsychotic medication (66/74; 89.2\%) vs none of the CHR participants $(0 / 45 ; 0 \%)$.

HC participants $(n=85)$ were recruited from the community and did not meet criteria for any Axis I diagnosis based on the SCID, or for participants $<16$ years of age, the Kiddie Schedule for Affective Disorders and Schizophrenia for School-Age Children, Present and Lifetime Version (Kaufman et al, 1997). Inclusion criteria across all participants required good physical health, English fluency, and a 12-35-year age range. Participants were excluded for DSM-IV past year substance dependence (excepting nicotine), a history of head injury with loss of consciousness, neurological illness, or, for HC participants, a first-degree relative with a psychotic illness. Written informed consent or assent was obtained from study participants under protocols approved by the institutional review board at UCSF.

\section{Data Acquisition and Processing}

Resting FMRI data were acquired on a Siemens 3T TIM TRIO scanner at UCSF Neuroimaging Center using a wholebrain echo-planar imaging (EPI) sequence with a 6-min acquisition time: 180 functional images were acquired (32 axial slices, $3.5 \mathrm{~mm}$ slice thickness, $1.05 \mathrm{~mm}$ interslice gap, $\mathrm{TR}=2 \mathrm{~s}, \mathrm{TE}=29 \mathrm{~ms}$, flip angle $=75^{\circ}, \mathrm{FOV}=24 \mathrm{~cm}$ ). Participants were instructed to rest with eyes closed and to stay awake.

Image preprocessing was performed with Statistical Parametric Mapping (SPM8: http://www.fil.ion.ucl.ac.uk/ $\mathrm{spm} / \mathrm{software} / \mathrm{spm} 8 /$ ). Images were motion-corrected via affine registration and slice time corrected. ACompCor, a regression-based algorithm for denoising BOLD data, was then applied (Behzadi et al, 2007). ACompCor performs a principal components analysis on time series data by 
deriving white matter and CSF noise regions from participants' segmented high-resolution T1-weighted anatomical images coregistered to their functional data. Resulting noise components are then entered as regressors, along with regressors to account for scanner nonequilibrium and drift effects (Whitfield-Gabrieli and Nieto-Castanon, 2012), in the first-level voxelwise regression model of the resting FMRI data for each subject.

Preprocessed/denoised data were subjected to a fast Fourier transformation. The square root of the power for each frequency was calculated, yielding amplitude values. To calculate voxelwise fractional ALFF (fALFF), the sum of the amplitude values in the 0.01 to $0.08 \mathrm{~Hz}$ low-frequency power range (ie, ALFF) was divided by the sum of the amplitudes over the entire detectable power spectrum $(0$ to $0.25 \mathrm{~Hz}$ ). Relative to ALFF, the normalized fALFF measure shows reduced influence of physiological noise (Zou et al, 2008) and is more sensitive to proband $v s$ control differences (Meda et al, 2015). The mean motion-corrected functional image was normalized to the Montreal Neurological Institute (MNI) EPI template using a 12-parameter affine transformation and $4 \times 5 \times 4$ nonlinear basis functions, and the resulting transformation matrix was applied to the fALFF images. Images were resliced to $3 \mathrm{~mm}^{3}$ voxel resolution and spatially smoothed with a 6-mm full width half maximum Gaussian filter.

\section{Data Analysis}

To control for expected brain maturation across the age range studied and age differences between clinical groups, normal maturation effects were statistically removed from the fALFF data. To do this, we modeled normal aging effects in the HC group and then calculated age-adjusted $z$-scores for all subjects based on the HC group age regression (see Fryer et al, 2013; Pfefferbaum et al, 1992):

\section{Age-adjusted $z$-score $=($ observed fALFF value - predicted fALFF value based on participant's age)/standard error of regression from HC age-regression model.}

Thus, a participant's age-adjusted $z$-score voxelwise map reflects the deviation in fALFF activity, expressed in SD units, from that expected for a healthy individual of the same age. All regions showing a significant effect of Group were evaluated for curvilinear effects of age within the HC group, and in no region did the quadratic age term account for significant variance in fALFF beyond the linear age effect $(0.14<p<0.79)$, supporting the application of linear regression to control effects of age.

Voxelwise ANOVA was then conducted on age-adjusted fALFF $z$-score maps. In order to correct for multiple comparisons, statistical significance was based on applying an initial voxel-level cluster-defining height threshold of $p<0.005$ (extent threshold $=5$ voxels) to the main effect of Group F-map followed by retaining clusters that met a familywise error (FWE) cluster-level correction, $p<0.05$. Mean fALFF values from FWE-corrected clusters showing a significant main effect of Group were extracted for all participants and subjected to Tukey-Kramer honest significant difference (HSD)-corrected post hoc tests to determine the pattern of pairwise group differences contributing to the omnibus effect. Scan head motion was compared between the groups via ANOVA by calculating the Average Motion Displacement (Van Dijk et al, 2012), defined as the root mean square (RMS) of the scan-to-scan change in $\mathrm{x}-\mathrm{y}-\mathrm{z}$ translation (in $\mathrm{mm}$ ) or roll-pitch-yaw rotation (in degrees) parameters. The impact of differences in head motion between the groups was examined by rerunning analyses with a covariate for the motion metrics (translational or rotational) that differed between groups. The effects of antipsychotic medication dosage within the ESZ group was examined by correlating chlorpromazine (CPZ) dosage equivalents (Woods, 2003) with extracted fALFF cluster means from each ESZ patient within regions showing a significant Group effect. Nonparametric correlations (Spearman's rho) were used because of the nonnormality of the CPZ variable. Next, means for all clusters showing a significant main effect of Group were extracted and examined between CHR participants who converted to a psychotic disorder within 24 months of study entry $(n=6) v s$ nonconverters $(n=18)$ who had been followed clinically for at least 24 months. Finally, voxelwise parametric correlation analyses were used to examine the relationship between fALFF and symptom severity within each clinical (CHR, ESZ) group, yielding correlation maps relating each symptom domain examined to fALFF. For the ESZ group, PANSS mean Positive, Negative, and General symptom subscales were examined. For the CHR group, mean SOPS Positive, Negative, and Disorganized symptom subscales were examined. In addition, we examined correlations with SOPS item P1 (Unusual Thought Content/Delusional Ideas) and P2 (Suspiciousness/Persecutory Ideas), as these items independently contribute to the prediction of conversion to psychosis in CHR individuals (Cannon et al, 2008). Correlation maps were thresholded at $p<0.005$ (extent threshold $=5$ voxels) and clusters were retained that met a FWE cluster-level correction, $p<0.05$.

\section{RESULTS}

\section{Participant Demographic and Clinical Data}

The groups did not differ on gender or handedness $(p>0.37)$. There was a significant main effect of Group $(p=0.04)$ on parental socioeconomic status (pSES) (Hollingshead and Redlich, 1958), explained by the ESZ group's lower pSES compared with the HC group $(p=0.047)$. Analysis of average brain image motion displacement revealed no significant Group effect on rotational movement but a significant Group effect $(p=0.009)$ on the translational movement, owing to the ESZ group moving significantly more than the HC group $(p=0.009)$. There was a statistical trend toward a Group effect on age $(p=0.07)$, with post hoc tests showing the HC group was older than the CHR group $(p=0.05)$, with the ESZ differing from neither group $(p=0.28)$. See Table 1 for group means on demographic measures, statistical tests of all pairwise group differences, and clinical characteristics of the patient groups.

\section{Group Differences in Amplitude of LFOs}

FWE-corrected clusters of age-adjusted fALFF values indicating a main effect of Group were detected in nine regions. For all regions, the CHR group had mean fALFF 
Table I Demographic and Clinical Data for Participants in the Healthy Control (HC), Clinical High Risk for Psychosis (CHR), and Early IIIness Schizophrenia (ESZ) Groups

\begin{tabular}{|c|c|c|c|}
\hline & $\begin{array}{c}\mathrm{HC} \\
\text { Mean } \pm \mathrm{SD}\end{array}$ & $\begin{array}{c}\text { CHR } \\
\text { Mean } \pm \text { SD }\end{array}$ & $\begin{array}{c}\text { ESZ } \\
\text { Mean } \pm \text { SD }\end{array}$ \\
\hline$N^{\mathrm{a}}$ & 85 & 45 & 74 \\
\hline Gender (\% male) & $60.00 . \%$ & $53.33 \%$ & $66.22 \%$ \\
\hline $\begin{array}{l}\text { *Age (years) } \\
\text { Range: } 12-35\end{array}$ & $22.65 \pm 6.45$ & $20.34 \pm 4.70$ & $21.89 \pm 4.22$ \\
\hline *Parental SES ${ }^{b}$ & $44.84 \pm 20.17$ & $54.78 \pm 16.77$ & $54.19 \pm 13.80$ \\
\hline Handedness (\% right-handed) & $92.94 \%$ & $86.67 \%$ & $91.89 \%$ \\
\hline $\begin{array}{l}\text { *Average Translational Motion } \\
\text { Displacement }(\mathrm{mm})^{c}\end{array}$ & $0.059 \pm 0.026$ & $0.062 \pm 0.031$ & $0.073 \pm 0.031$ \\
\hline $\begin{array}{l}\text { Average Rotational Motion } \\
\text { Displacement (degrees) }\end{array}$ & $0.038 \pm 0.019$ & $0.043 \pm 0.022$ & $0.042 \pm 0.021$ \\
\hline Chlorpromazine equivalents (mg) & - & - & $276.11 \pm 341.88$ \\
\hline \multicolumn{4}{|l|}{$\begin{array}{l}\text { Total symptom ratings for patient } \\
\text { groups }\end{array}$} \\
\hline SOPS Positive & - & $9.33 \pm 4.17$ & - \\
\hline SOPS Negative & - & $11.63 \pm 5.94$ & - \\
\hline SOPS Disorganized & - & $5.44 \pm 3.38$ & - \\
\hline SOPS General & - & $8.68 \pm 4.56$ & - \\
\hline PANSS Positive & - & - & $13.86 \pm 4.75$ \\
\hline PANSS Negative & - & - & $17.25 \pm 6.55$ \\
\hline PANSS General & - & - & $33.31 \pm 8.73$ \\
\hline $\begin{array}{l}\% \text { Antipsychotic medication (\% } \\
\text { typical; atypical; unknown; none) }\end{array}$ & - & - & $\begin{array}{l}2.7 \% ; 81.1 \% ; \\
5.4 \% ; 10.8 \%\end{array}$ \\
\hline
\end{tabular}

*Significant omnibus test, $p<0.05$ :

*Age (trend toward significance): $F(2,20 I)=2.73, p=0.07$; Tukey-Kramer HSD post hoc tests $\mathrm{HC}>\mathrm{CHR}, p=0.05$;

*PSES: $F(2,202)=3.29, p=0.04$; Tukey-Kramer HSD post hoc tests, $\mathrm{HC}<$ ESZ, $p=0.05$.

*Average Translational Motion Displacement: $F(2,20 I)=4.78, p=0.009$;

Tukey-Kramer HSD post hoc tests HC $<$ ESZ, $p=0.009$.

Nonsignificant $(p>0.05)$ comparisons:

Gender: $\chi^{2}(2, N=204)=1.99, p=0.37$;

Handedness: $\chi^{2}(2, N=204)=1.51, p=0.47$;

Average Rotational Motion Displacement: $F(2,20 I)=1.26, p=0.29$

aSymptom ratings within 30 days of MR scan not available for I ESZ participant: all CHR patients had positive symptom SOPS ratings but 6 were missing disorganized and general symptom ratings and 7 were missing negative symptom ratings because of incomplete assessment sessions.

${ }^{b}$ Parental socioeconomic status (PSES) measured by the Hollingshead 2-Factor Index. Higher Hollingshead scores indicate lower pSES. All other assessment measures are scaled such that higher scores reflect greater levels of the measured variable.

'Average Motion Displacement defined by Van Dijk et al (20/2) as the root mean square (RMS) of the scan-to-scan change in the $x-y-z$ translation (in $\mathrm{mm}$ ) or roll-pitch-yaw rotation (in degrees) parameters.

values that were in between $\mathrm{HC}$ values and the lower amplitudes observed in ESZ patients (ie, $\mathrm{HC}>\mathrm{CHR}>\mathrm{ESZ}$ ). See Figure 1 and Table 2 for mean fALFF cluster values and test statistics for pairwise group follow-up tests. Results are reported below for each regional cluster, including Tukey HSD-corrected pairwise follow-up comparisons. See Supplementary Information for depiction of full extent of voxelwise findings, plots of cluster means unadjusted for age, and results and plots of additional analyses examining subcortical regions of interest.
Bilateral temporoparietooccipital/posterior cingulate cortex $(P C C)$. This posterior cortical cluster contained mostly left-sided medial parietoocciptal cortex (including bilateral cuneus, precuneus, PCC, and lingual gyri), extending to the temporal lobe to include parahippocampal gyrus. fALFF was marginally reduced in the CHR relative to $\mathrm{HC}(p=0.06)$ group, and reduced in the ESZ group relative to both CHR $(p=0.01)$ and $\mathrm{HC}(p<0.001)$ groups.

Precuneus/PCC. This cluster contained bilateral precuneus and PCC. fALFF was reduced in the CHR $(p=0.01)$ and ESZ $(p<0.001)$ groups relative to the HC group.

Left inferior parietal lobule (IPL). This left hemispheric IPL cluster included Brodmann area (BA) 40. fALFF values were reduced in the ESZ group relative to both HC $(p<0.001)$ and CHR $(p=0.002)$ groups.

Left temporoparietal junction (TPJ). This left hemispheric TPJ cluster contained supramarginal gyrus and BA 39, and extended inferiorly to the superior and middle temporal gyri. fALFF values were reduced in the CHR $(p=0.002)$ and ESZ $(p<0.001)$ groups relative to the HC group.

Right inferior parietal lobule. This right hemispheric IPL cluster included BA 40. FALFF values were reduced in the CHR $(p=0.007)$ and ESZ $(p<0.001)$ groups relative to the $\mathrm{HC}$ group.

Right anterior inferior parietal lobule. This right hemispheric anterior IPL cluster was anterolateral to the right IPL cluster. It overlapped with BA 40, extending slightly into pre- and post-central gyri. fALFF values were reduced in the CHR $(p=0.007)$ and ESZ $(p<0.001)$ groups relative to the $\mathrm{HC}$ group.

Right parietooccipital cortex. This right hemispheric cluster contained parietooccipital cortex, including precuneus and cuneus. fALFF values were reduced in the CHR $(p=0.009)$ and ESZ $(p<0.001)$ groups relative to the HC group.

Left cerebellum. This cluster encompassed the left posterior cerebellar lobe. fALFF values were reduced in the CHR $(p=0.001)$ and ESZ $(p<0.001)$ groups relative to the HC group.

Midline cerebellum. This midline cerebellar cluster included posterior vermis. fALFF values were reduced in the CHR $(p=0.009)$ and ESZ $(p<0.001)$ groups relative to the $\mathrm{HC}$ group.

For all regions, unless reported otherwise, the CHR and ESZ groups did not differ from each other $(p>0.1)$. In addition, lower fALFF in the ESZ relative to HC group was also observed in the thalamus and caudate based on regionof-interest analysis (see Supplementary Information), although effects were of smaller magnitude than those reported in the primary whole-brain voxelwise analysis. 


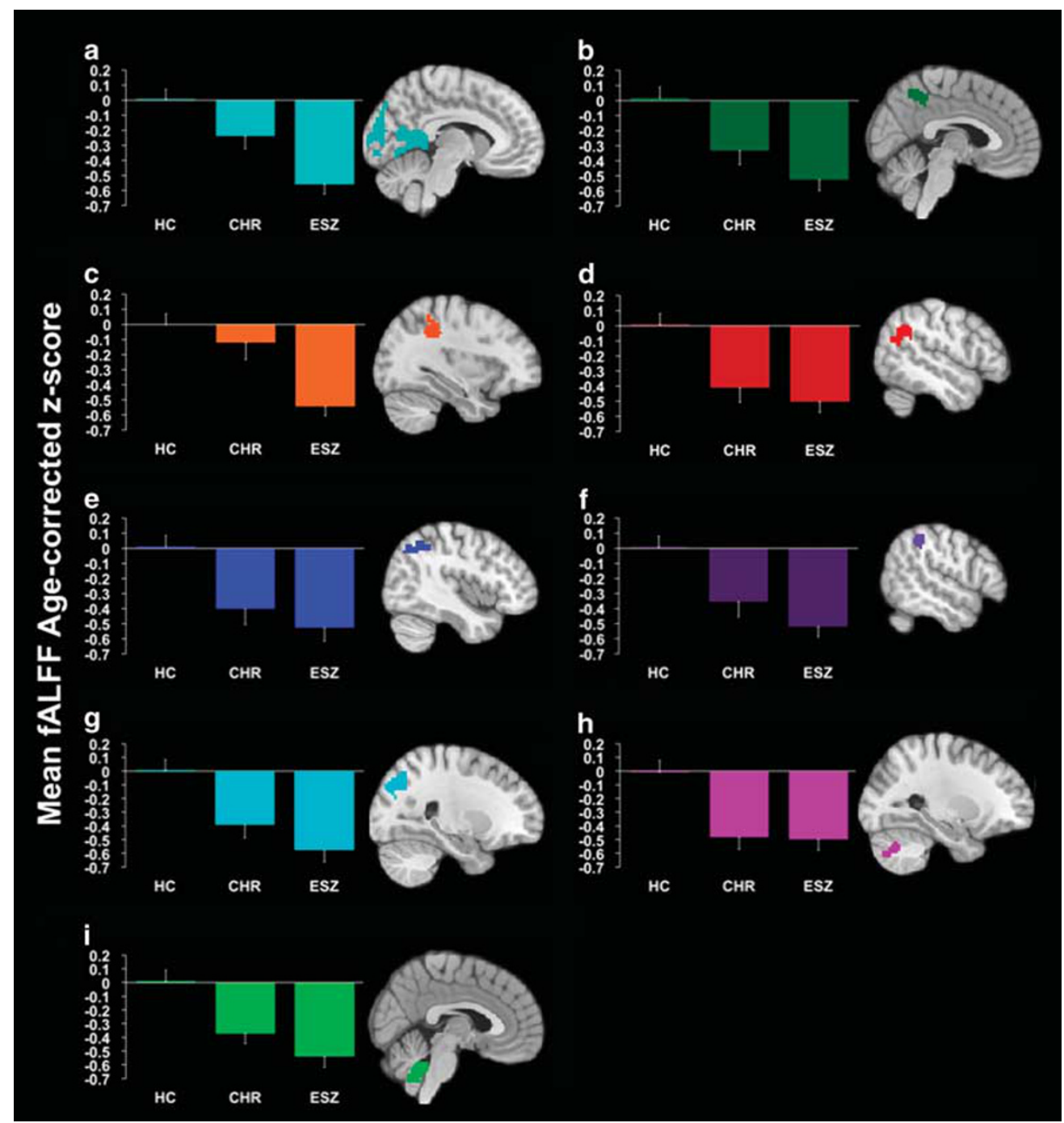

Figure I Bar graphs display age-adjusted z-score cluster mean ( \pm SEM) fractional amplitude of low-frequency fluctuations (fALFF) by Group for each cluster showing a significant ( $p<0.005$ height threshold; $p<0.05$ familywise error FWE cluster-corrected) main effect of Group. Regions correspond to cluster labels reported in Results section: (a) Bilateral temporoparietooccipital cortex/posterior cingulate cortex (PCC), (b) precuneus/PCC, (c) left inferior parietal lobule, (d) left temporoparietal junction, (e) right inferior parietal lobule, (f) right anterior inferior parietal lobule, (g) right parietooccipital cortex, (h) left cerebellum, and (i) cerebellum midline. Age-corrected z-scores are based on age relationships modeled in healthy control (HC) group (see text). Accordingly, HC means $=0$

\section{Consideration of Motion Artifact and Medication Dosage on Group Effects}

Regional fALFF clusters showing a significant main effect of Group were extracted for all subjects and analyses were repeated adding translational displacement as a covariate. All group magnitude differences reported in the original analysis remained significant (main effect of Group p-values all $<0.001)$. Corrected pairwise comparisons also revealed the same pattern of significance $(p<0.05)$, suggesting that observed effects were not explained by group differences in motion. Next, we examined the effects of antipsychotic medication dosage on AALFF within our ESZ patient sample. $\mathrm{CPZ}$ dosage did not correlate with mean fALFF within any of the regions showing a main effect of Group (all Spearman's rho $<|0.16|$; all $p>0.20$ ).

\section{Conversion Effects with CHR Group}

Within the CHR group, there were no differences on extracted cluster means for any of the 9 regions showing a main effect of Group between individuals with longitudinal follow-up data who converted to a psychotic disorder $(n=6)$ $v s$ those who did not $(n=18)$ within a 24 -month period of clinical follow-up $(0.27<p<0.97)$.

\section{Correlation of LFO Amplitude with Clinical Symptoms}

CHR group. Within the CHR group, voxelwise correlations between fALFF and mean SOPS clinical ratings revealed several significant associations (see Table 3 and Figure 2). No significant associations were observed between fALFF and 
Table 2 Brain Regions Showing Significant ${ }^{a}$ Group Differences in fALFF When Comparing Age-Adjusted Z-Scores of Healthy Control (HC), Clinical High Risk for Psychosis (CHR), and Early Illness Schizophrenia (ESZ) Groups (N=204)

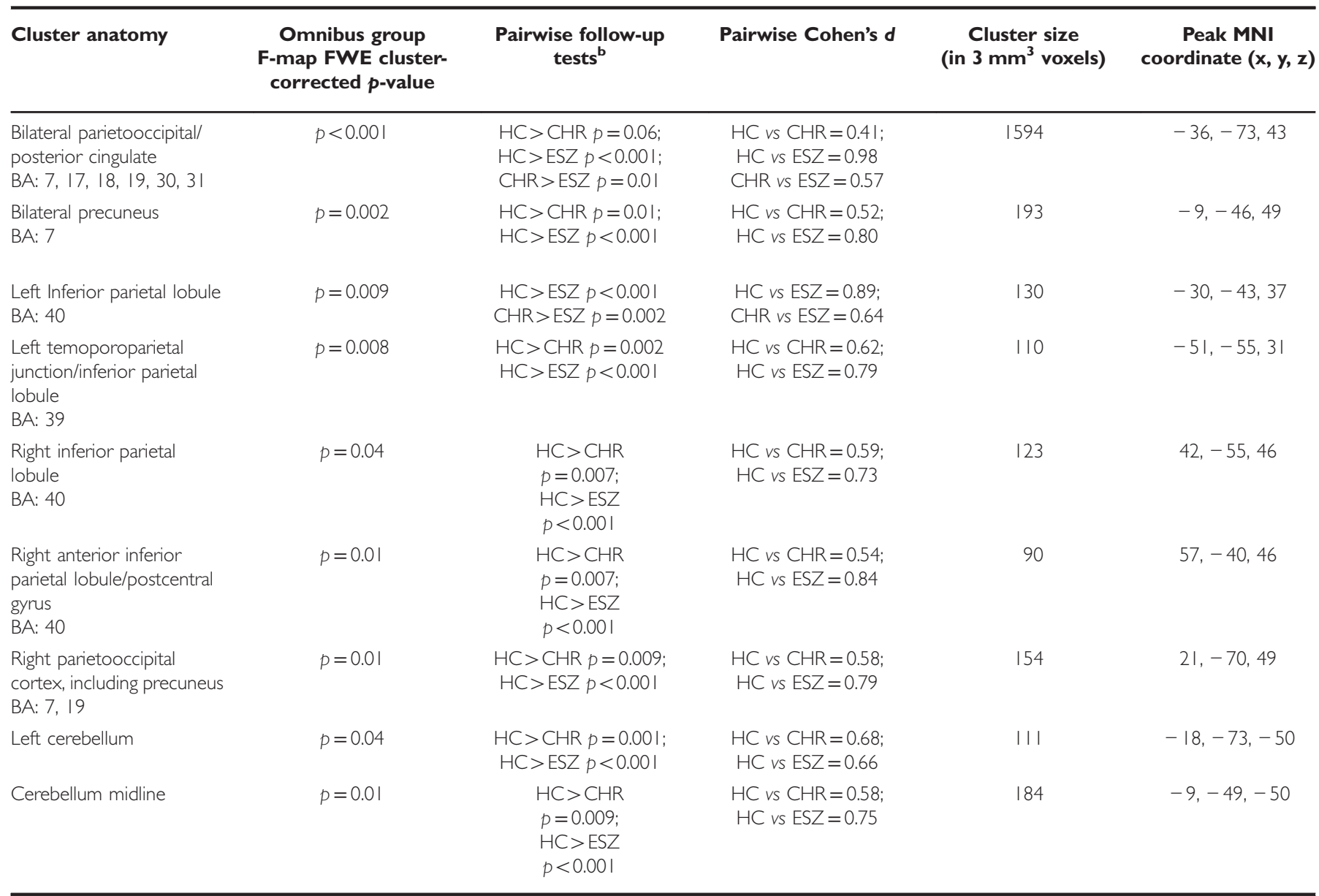

Abbreviation: BA, Brodmann area.

${ }^{a}$ Cluster-corrected $p$-values are familywise error (FWE) corrected at a height threshold of $p<0.005$.

${ }^{\text {b}}$ Tukey-Kramer HSD post hoc tests, two tailed, $p<0.05$.

Table 3 Brain Regions Showing a Significant ${ }^{a}$ Correlation between fALFF and Symptom Severity within the Clinical High Risk for Psychosis $(\mathrm{CHR})$ Group

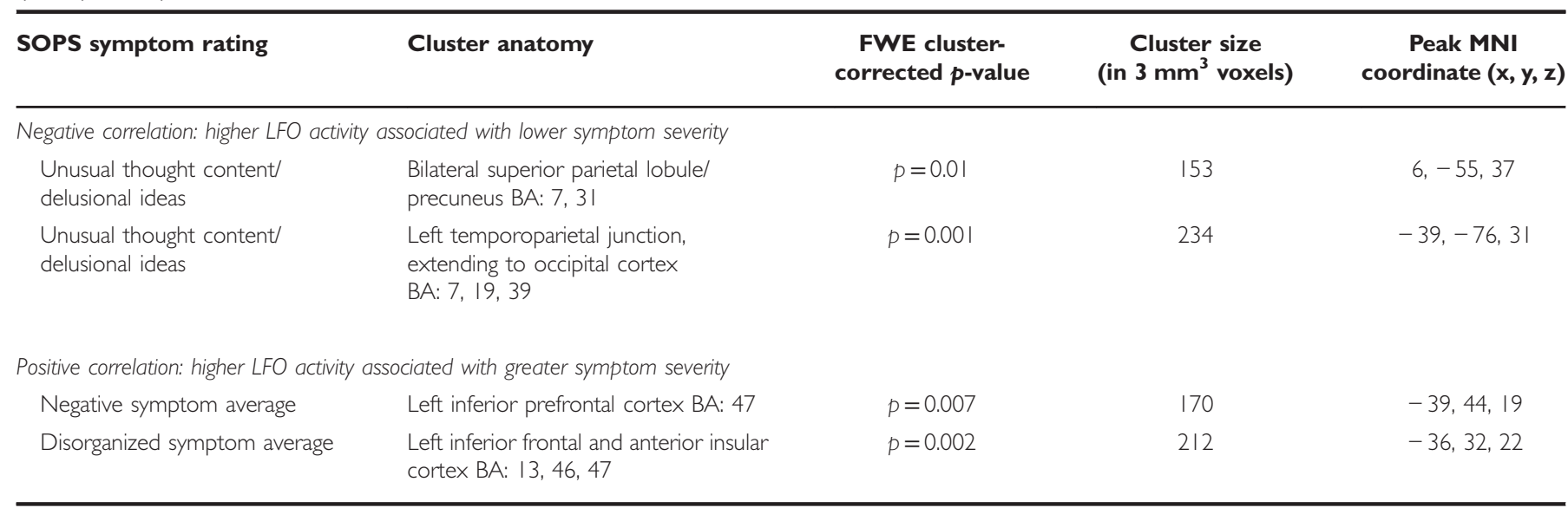

Abbreviation: BA, Brodmann area.

${ }^{a}$ Cluster-corrected $p$-values are familywise error (FWE) corrected and based on a cluster-defining height threshold of $p<0.005$. 

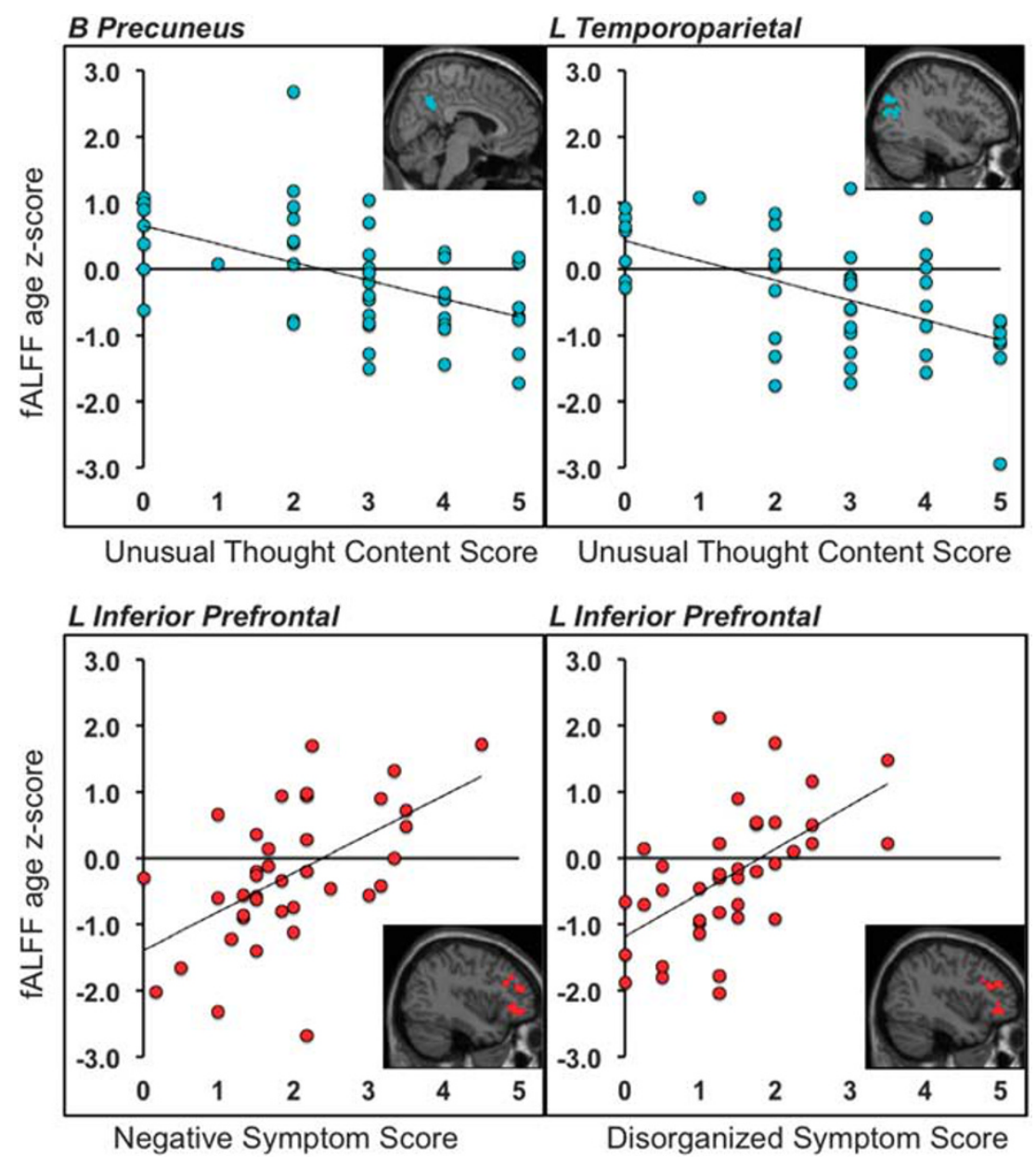

Figure 2 Correlation maps showing significant ( $p<0.005$ height threshold; $p<0.05$ FWE cluster-corrected) associations between clinical symptom severity and fALFF within the clinical high risk for psychosis (CHR) group. The scatterplot and regression line are shown for the peak voxel within the cluster to illustrate the effect. Clinical scores are from the Scale of Prodromal Symptoms (SOPS).

the Positive symptom subscale, or item P2 (Suspiciousness/ Persecutory Ideas).

Negative correlations. For SOPS item P1 (Unusual Thought Content/Delusional Ideas), we observed two regions with significant negative correlations (ie, lower fALFF associated with more severe symptoms): one in medial superior parietal cortex and the other in left TPJ. The medial superior parietal region was bilateral, although it contained mostly left-sided precuneus and superior parietal lobule. The left TPJ region contained primarily middle temporal gyrus and precuneus and extended into occipital cortex. Both of the regions showing a negative correlation overlapped slightly with regions showing a main effect of group in fALFF.

Positive correlations. Significant positive correlations were observed between fALFF and SOPS Negative and Disorganized symptom subscales. For the Negative Symptom subscale, greater symptom severity was associated with higher fALFF in a left prefrontal cortical cluster encompassing middle and inferior frontal gyri. For the Disorganized Symptom subscale, a significant positive correlation cluster emerged in the left prefrontal and anterior insular cortex that was larger than, but overlapping with, the cluster showing a positive correlation with the Negative Symptom subscale. There was no overlap between regions showing a positive correlation (ie, higher fALFF related to higher symptom levels) and regions showing a main effect of group in fALFF.

ESZ group. Within the ESZ group, we found no significant correlations between fALFF and PANSS Positive, Negative, or General subscales. However, when examining the distribution of PANSS variables, we noted that a majority of items had a mode of 1 (indicating that the symptom was not present for the majority of individuals) and it was evident that PANSS items were more skewed than SOPS items in our sample (mean PANSS item skewness 1.12 \pm .97 , range $=0.02$ to $4.34 \mathrm{vs}$ mean SOPS item skewness $0.68 \pm 0.58$, range $=-0.45$ to 1.66 ). Because of concerns about the appropriateness of conducting correlational analyses on PANSS subscales that included many skewed items, we instead conducted correlation analyses with individual PANSS items that met the following criteria: mode $>2$, skewness $<25$ th percentile of all items. Out of 30 PANSS items, 4 met these distributional requirements: Attention, Anxiety, Emotional Withdrawal, and 
Table 4 Brain Regions Showing a Significant ${ }^{a}$ Correlation between fALFF and Symptom Severity within the Early IIIness Schizophrenia (ESZ) Group

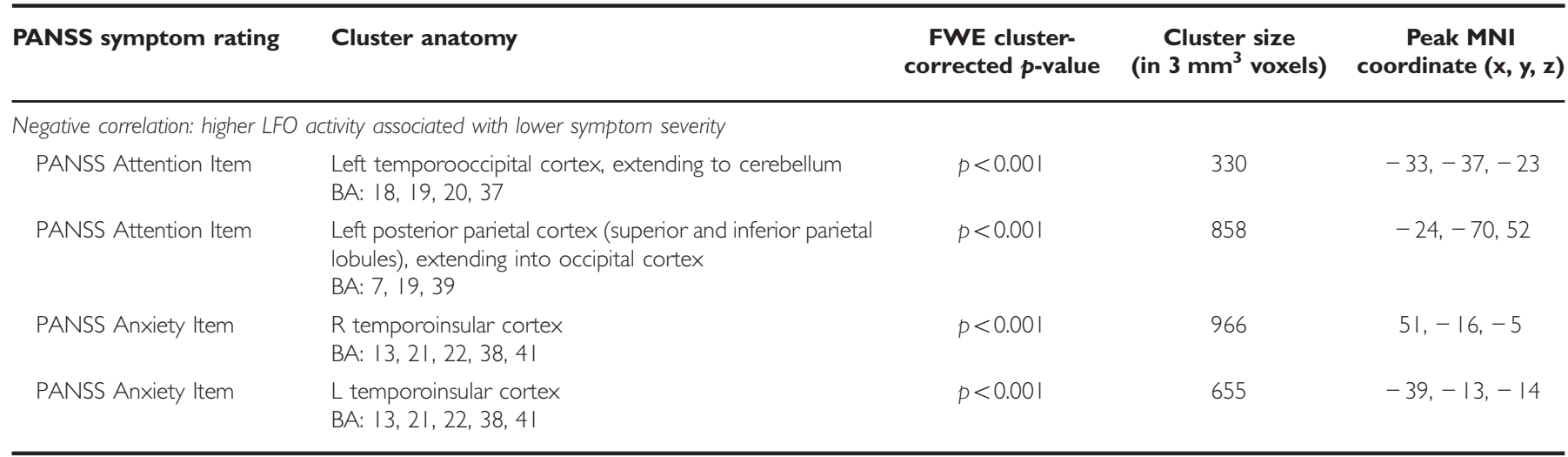

Abbreviation: BA, Brodmann area.

${ }^{a}$ Cluster-corrected $p$-values are familywise error (FWE) corrected and based on a cluster-defining height threshold of $p<0.005$.
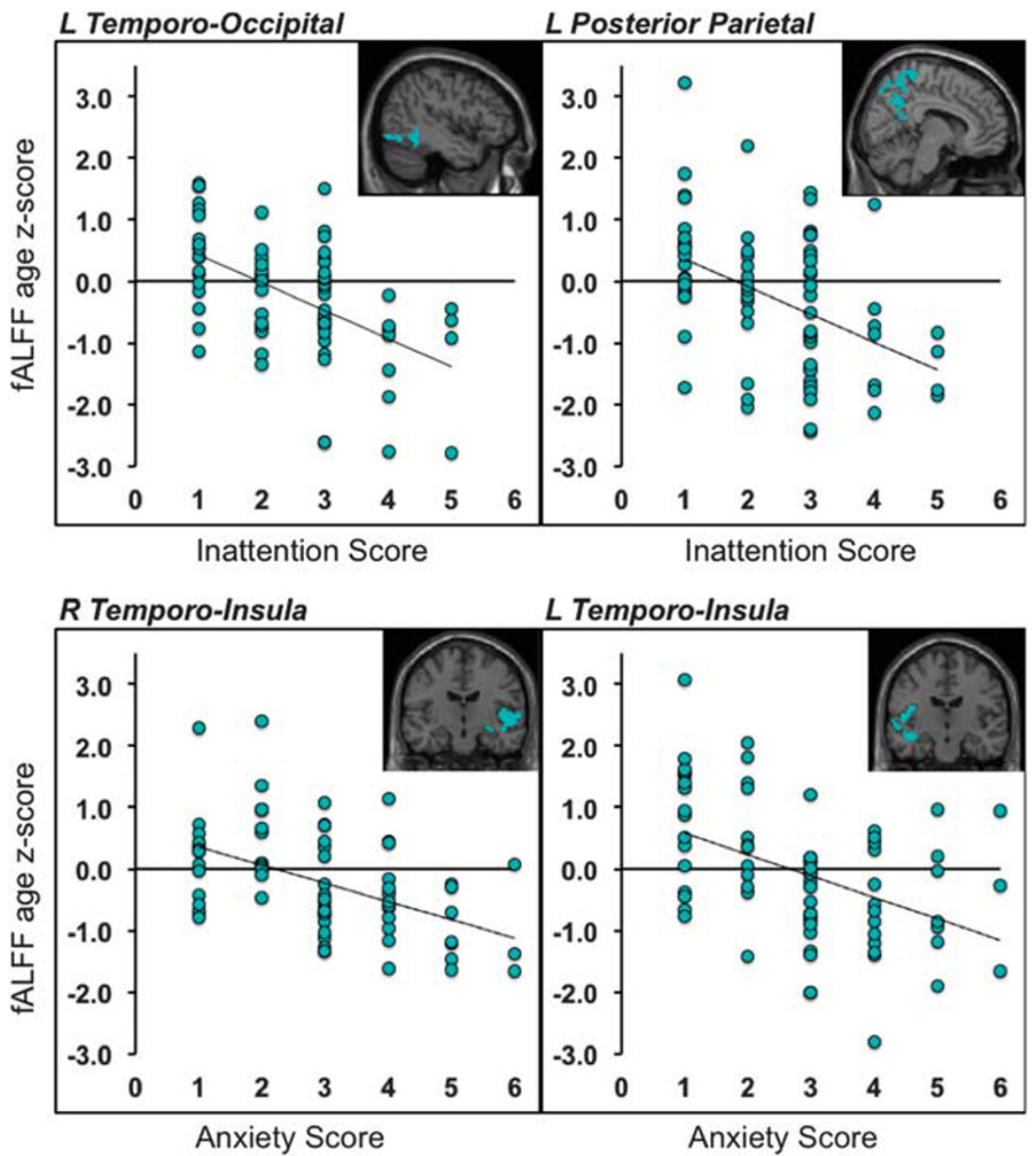

Figure 3 Correlation maps showing significant ( $p<0.005$ height threshold; $p<0.05$ FWE cluster-corrected) associations between clinical symptom severity and fALFF within the early illness schizophrenia (ESZ) patient group. Clinical scores are from the Positive and Negative Syndrome Scale (PANSS).

Passive/Apathetic Social Withdrawal. No correlations were identified with either the Emotional or Passive/Apathetic Social Withdrawal items, but significant associations were identified between LFO activity and Attention and Anxiety items.
Negative correlations. We observed two significant regions with negative correlations in the left posterior parietal and left temporooccipital cortices for which lower LFO activity related to higher inattention symptoms (see Table 4 and Figure 3). Both of the inattention 
correlation clusters overlapped with regions showing a main effect of Group in fALFF. In addition we observed two significant negative correlation clusters in bilateral temporoinsular cortex for which lower LFO activity related to higher anxiety levels. The left-sided anxiety correlation cluster overlapped with regions showing a main effect of Group in fALFF.

Positive correlations. There were no positive correlations identified, indicating that for no regions examined did higher LFO activity relate to higher symptom levels.

\section{DISCUSSION}

We identified a number of posterior cortical and cerebellar regions where the strength of resting LFO BOLD activity oscillations differed across CHR, ESZ, and HC groups. Within all regions revealing a significant voxelwise main effect of Group, CHR individuals had LFO amplitudes intermediate between the HC and ESZ groups (ie, HC $>$ CHR $>$ ESZ), consistent with a potentially 'prodromal' effect (see Figure 1). Similarly, pairwise effect sizes of reduced LFO amplitude, relative to the HC group, were larger (average Cohen's $d=0.80$ ) and ranged from 'medium to large' effect sizes in the ESZ patient group compared with the reductions in the CHR group (average Cohen's $d=0.52$ ) that ranged from 'small to medium' effect sizes (Cohen, 1988). Examination of subcortical (thalamus, caudate, putamen) regions of interest (see Supplementary Information) yielded borderline significant between-group differences between the ESZ and HC groups in the caudate and thalamus. Subcortical group effects were of considerably smaller magnitude than those observed in the primary voxelwise analysis and did not involve the CHR group.

These results extend prior findings of LFO amplitude alterations in schizophrenia by demonstrating that regional amplitude abnormalities are present, but attenuated, in individuals at elevated clinical risk for psychosis.

For both clinical groups, LFO strength was associated with symptoms (see Figures 2 and 3). Within the ESZ patient group, smaller LFO amplitudes in left temporooccipital and posterior parietal cortex and bilateral temporal-insular cortex related to higher levels of inattention and anxiety, respectively. Within the CHR group, lower LFO activity in bilateral superior parietal lobule, precuneus, and left TPJ related to higher levels of unusual thought content. Unusual thought content is one of the several clinical risk factors shown to contribute uniquely to psychosis conversion risk (Cannon et al, 2008). For both ESZ and CHR groups, most of the regions showing negative correlations with symptoms partially overlapped with regions showing significant reductions in fALFF relative to the HC group (see Supplementary Information), lending clinical relevance to these fALFF reductions. Finally, increased LFO amplitude was associated with more severe disorganized and negative symptoms in the CHR group in left inferior prefrontal cortex. Importantly, this PFC region is anatomically distinct from posterior regions showing negative correlations between LFO amplitude and symptom severity and also did not show LFO amplitude differences between the groups.
We did not observe LFO amplitude differences between those CHR individuals who subsequently converted to a psychotic disorder $v s$ those who did not during a 24-month clinical follow-up period. Owing to the relatively small sample of longitudinal follow-up data, and particularly the small number of converters, these null findings must be interpreted with caution. Therefore, an important future research direction is to identify what aspects of clinical prognosis (eg, adaptive functioning decrements, distress levels, conversion to psychosis) do the observed alterations in regional LFO amplitude relate to. LFO amplitudes were reduced in first-degree relatives of schizophrenia patients in some (Guo et al, 2015; Tang et al, 2015), but not all (Lui et al, 2015; Meda et al, 2015), prior studies, suggesting that LFO amplitude deficits may be related to genetic risk for schizophrenia. Regardless, our findings suggest that intrinsic BOLD LFO functioning relates to clinical risk for psychosis, and furthermore may represent an illness vulnerability marker that worsens after illness onset.

The potential role of LFO activity in gating or maintaining functional connectivity likely has particular relevance for understanding schizophrenia pathophysiology, given the prominence of large-scale connectivity to conceptual understandings of the disorder (Andreasen, 1999; Friston, 1998). With regard to illness risk and conversion, functional dysconnectivity may be a final pathological consequence of aggregated genetic and environmental vulnerabilities that confer risk toward developing schizophrenia (Cannon, 2015). Dysconnectivity of intrinsic functioning has been identified in samples of CHR youth, including patterns of aberrant default mode network (Shim et al, 2010), prefrontal-thalamic and thalamic-sensorimotor (Anticevic et al, 2015) connectivity. Limited research has examined schizophrenia-related functional connectivity measures in light of LFO function. However, one small study found that areas of frontoparietal and occipitoparietal hypoconnectivity in schizophrenia showed reduced intraregional homogeneity and power spectral density, suggesting that functional dysconnectivity may reflect local LFO signal reduction and reduced signal-to-noise ratio (Zalesky et al, 2012).

This study is limited by insufficient longitudinal follow-up data to permit well-powered comparisons of CHR individuals who subsequently converted to a psychotic illness $v s$ those who did not. This limits inferences that we can make regarding whether the LFO amplitude reductions observed in our CHR group reflect an intermediate level of abnormality that progresses during the transition to psychosis or a fullblown abnormality evident only in the subgroup of high-risk patients who later develop a psychotic disorder. Future studies employing a longitudinal design across the illness course from clinical high-risk states to conversion to psychosis are needed to address this important question. In addition, antipsychotic medication status differed between the groups. However, analysis of medication effects on LFO magnitude within the medicated ESZ patients was not consistent with dosage accounting for observed effects, and the presence of LFO magnitude alterations within antipsychotic-naive CHR individuals further argues against medication effects accounting for the fALFF abnormalities observed in the ESZ patients. Furthermore, the previous literature is inconsistent regarding the direction and anatomical distribution of resting LFO alterations in 
schizophrenia. To the extent that these inconsistencies result from sampling error and insufficient power common in smaller studies, they may be clarified through further research with larger samples. However, prior inconsistencies may also validly reflect clinical, neurocognitive, and pathophysiological heterogeneity in schizophrenia, with results varying depending on how this heterogeneity is represented in any given patient sample. Therefore, there is a need to examine the relationships between resting LFO and clinical and neurocognitive functioning in patients and healthy individuals, rather than assuming that these functional domains are consistently represented across study samples. Despite these limitations, our results suggest that the amplitude of intrinsic LFO activity, which is easily and reliably measureable with resting-state FMRI, is modulated by illness stage and covaries with clinical symptoms. Our findings of similar but less pronounced LFO amplitude reductions in prospectively identified, antipsychotic medication-free CHR youth add to a growing body of literature indicating that intrinsic functional activity alterations associated with schizophrenia predate psychosis onset.

\section{FUNDING AND DISCLOSURE}

Within the past three years, Dr Mathalon has received compensation as a consultant for Boehringer-Ingelheim, Amgen, and Hoffman-LaRoche. The other authors declare no conflict of interest.

\section{ACKNOWLEDGMENTS}

This research was supported by NIMH: MH076989 (to DH Mathalon) and VA: CX001028 (to SL Fryer). We thank Nicholas Diaz for assistance with manuscript preparation.

\section{REFERENCES}

Andreasen NC (1999). A unitary model of schizophrenia: Bleuler's 'fragmented phrene' as schizencephaly. Arch Gen Psychiatry 56: 781-787.

Anticevic A, Haut K, Murray JD, Repovs G, Yang GJ, Diehl C et al (2015). Association of thalamic dysconnectivity and conversion to psychosis in youth and young adults at elevated clinical risk. JAMA Psychiatry 72: 882-891.

Behzadi Y, Restom K, Liau J, Liu TT (2007). A component based noise correction method (CompCor) for BOLD and perfusion based fMRI. Neuroimage 37: 90-101.

Biswal B, Yetkin FZ, Haughton VM, Hyde JS (1995). Functional connectivity in the motor cortex of resting human brain using echo-planar MRI. Magn Reson Med 34: 537-541.

Buzsáki G, Logothetis N, Singer W (2013). Scaling brain size, keeping timing: evolutionary preservation of brain rhythms. Neuron 80: 751-764.

Cannon TD (2015). Network dysconnectivity: a psychosistriggering mechanism? Biol Psychiatry 77: 927-928.

Cannon TD, Cadenhead K, Cornblatt B, Woods SW, Addington J, Walker E et al (2008). Prediction of psychosis in youth at high clinical risk: a multisite longitudinal study in North America. Arch Gen Psychiatry 65: 28-37.

Cohen J (1988). Statistical Power Analysis for the Behavioral Sciences, 2nd edn. Laurence Erlbaum Associates: Hillsdale, NJ, $567 \mathrm{pp}$.
First MB, Spitzer RL, Gibbon M, Williams JB (2002). Structured Clinical Interview for DSM-IV-TR Axis I Disorders, Research Version, Patient Edition (SCID-I/P). Biometrics Research, New York State Psychiatric Institute: New York.

Fox MD, Snyder AZ, Vincent JL, Raichle ME (2007). Intrinsic fluctuations within cortical systems account for intertrial variability in human behavior. Neuron 56: 171-184.

Friston KJ (1998). The disconnection hypothesis. Schizophr Res 30: 115-125.

Fryer SL, Roach BJ, Ford JM, Turner JA, van Erp TG, Voyvodic J et al (2015). Relating intrinsic low-frequency BOLD cortical oscillations to cognition in schizophrenia. Neuropsychopharmacology 40: 2705-2714.

Fryer SL, Woods SW, Kiehl KA, Calhoun VD, Pearlson GD, Roach BJ et al (2013). Deficient suppression of default mode regions during working memory in individuals with early psychosis and at clinical high-risk for psychosis. Front Psychiatry 4: 92.

Guo W, Song Y, Liu F, Zhang Z, Zhang J, Yu M et al (2015). Dissociation of functional and anatomical brain abnormalities in unaffected siblings of schizophrenia patients. Clin Neurophysiol 126: 927-932.

He BJ, Snyder AZ, Zempel JM, Smyth MD, Raichle ME (2008). Electrophysiological correlates of the brain's intrinsic large-scale functional architecture. Proc Natl Acad Sci USA 105: 16039-16044.

He Z, Deng W, Li M, Chen Z, Jiang L, Wang Q et al (2013). Aberrant intrinsic brain activity and cognitive deficit in firstepisode treatment-naive patients with schizophrenia. Psychol Med 43: 769-780.

Hiltunen T, Kantola J, Abou Elseoud A, Lepola P, Suominen K, Starck $\mathrm{T}$ et al (2014). Infra-slow EEG fluctuations are correlated with resting-state network dynamics in fMRI. J Neurosci 34: 356-362.

Hollingshead A, Redlich F (1958). Social Class and Mental Illness. John Wiley and Sons: New York.

Hoptman MJ, Zuo XN, Butler PD, Javitt DC, D'Angelo D, Mauro CJ et al (2010). Amplitude of low-frequency oscillations in schizophrenia: a resting state fMRI study. Schizophr Res 117: 13-20.

Huang XQ, Lui S, Deng W, Chan RC, Wu QZ, Jiang LJ et al (2010). Localization of cerebral functional deficits in treatment-naive, first-episode schizophrenia using resting-state fMRI. Neuroimage 49: 2901-2906.

Kaufman J, Birmaher B, Brent D, Rao U, Flynn C, Moreci P et al (1997). Schedule for Affective Disorders and Schizophrenia for School-Age Children-Present and Lifetime Version (K-SADSPL): initial reliability and validity data. J Am Acad Child Adolesc Psychiatry 36: 980-988.

Kay SR, Fiszbein A, Opler LA (1987). The positive and negative syndrome scale (PANSS) for schizophrenia. Schizophr Bull 13: 261-276.

Leopold DA, Murayama Y, Logothetis NK (2003). Very slow activity fluctuations in monkey visual cortex: implications for functional brain imaging. Cereb Cortex 13: 422-433.

Lui S, Li T, Deng W, Jiang L, Wu Q, Tang H et al (2010). Short-term effects of antipsychotic treatment on cerebral function in drug-naive first-episode schizophrenia revealed by 'resting state' functional magnetic resonance imaging. Arch Gen Psychiatry 67: 783-792.

Lui S, Yao L, Xiao Y, Keedy SK, Reilly JL, Keefe RS et al (2015). Resting-state brain function in schizophrenia and psychotic bipolar probands and their first-degree relatives. Psychol Med 45: 97-108.

Mathalon DH, Sohal VS (2015). Neural oscillations and synchrony in brain dysfunction and neuropsychiatric disorders: it's about time. JAMA Psychiatry 72: 840-844.

Meda SA, Wang Z, Ivleva EI, Poudyal G, Keshavan MS, Tamminga CA et al (2015). Frequency-specific neural signatures of spontaneous low-frequency resting state fluctuations in psychosis: evidence from Bipolar-Schizophrenia Network on Intermediate Phenotypes (B-SNIP) Consortium. Schizophr Bull 41: 1336-1348. 
Miller TJ, McGlashan TH, Rosen JL, Cadenhead K, Cannon T, Ventura J et al (2003). Prodromal assessment with the structured interview for prodromal syndromes and the scale of prodromal symptoms: predictive validity, interrater reliability, and training to reliability. Schizophr Bull 29: 703-715.

Miller TJ, McGlashan TH, Rosen JL, Somjee L, Markovich PJ, Stein K et al (2002). Prospective diagnosis of the initial prodrome for schizophrenia based on the Structured Interview for Prodromal Syndromes: preliminary evidence of interrater reliability and predictive validity. Am J Psychiatry 159: 863-865.

Narr KL, Leaver AM (2015). Connectome and schizophrenia. Curr Opin Psychiatry 28: 229-235.

Palva JM, Palva S (2012). Infra-slow fluctuations in electrophysiological recordings, blood-oxygenation-level-dependent signals, and psychophysical time series. Neuroimage 62: 2201-2211.

Pfefferbaum A, Lim KO, Zipursky RB, Mathalon DH, Rosenbloom MJ, Lane B et al (1992). Brain gray and white matter volume loss accelerates with aging in chronic alcoholics: a quantitative MRI study. Alcohol Clin Exp Res 16: 1078-1089.

Ren W, Lui S, Deng W, Li F, Li M, Huang X et al (2013). Anatomical and functional brain abnormalities in drug-naive first-episode schizophrenia. Am J Psychiatry 170: 1308-1316.

Roach BJ, Mathalon DH (2008). Event-related EEG time-frequency analysis: an overview of measures and an analysis of early gamma band phase locking in schizophrenia. Schizophr Bull 34: 907-926.

Shim G, Oh JS, Jung WH, Jang JH, Choi CH, Kim E et al (2010). Altered resting-state connectivity in subjects at ultra-high risk for psychosis: an fMRI study. Behav Brain Funct 6: 58.

Sui J, Pearlson GD, Du Y, Yu Q, Jones TR, Chen J et al (2015). In search of multimodal neuroimaging biomarkers of cognitive deficits in schizophrenia. Biol Psychiatry 78: 794-804.

Tang Y, Chen K, Zhou Y, Liu J, Wang Y, Driesen N et al (2015). Neural activity changes in unaffected children of patients with schizophrenia: a resting-state fMRI study. Schizophr Res 168: 360-365.

Turner JA, Damaraju E, van Erp TG, Mathalon DH, Ford JM, Voyvodic J et al (2013). A multi-site resting state fMRI study on the amplitude of low frequency fluctuations in schizophrenia. Front Neurosci 7: 137.
Uhlhaas PJ, Haenschel C, Nikolic D, Singer W (2008). The role of oscillations and synchrony in cortical networks and their putative relevance for the pathophysiology of schizophrenia. Schizophr Bull 34: 927-943.

Van Dijk KR, Sabuncu MR, Buckner RL (2012). The influence of head motion on intrinsic functional connectivity MRI. Neuroimage 59: 431-438.

Weinberger DR (1987). Implications of normal brain development for the pathogenesis of schizophrenia. Arch Gen Psychiatry 44: 660-669.

Whitfield-Gabrieli S, Nieto-Castanon A (2012). Conn: a functional connectivity toolbox for correlated and anticorrelated brain networks. Brain Connect 2: 125-141.

Woods SW (2003). Chlorpromazine equivalent doses for the newer atypical antipsychotics. J Clin Psychiatry 64: 663-667.

Woods SW, Addington J, Cadenhead KS, Cannon TD, Cornblatt BA, Heinssen R et al (2009). Validity of the prodromal risk syndrome for first psychosis: findings from the North American Prodrome Longitudinal Study. Schizophr Bull 35: 894-908.

Xu Y, Zhuo C, Qin W, Zhu J, Yu C (2015). Altered spontaneous brain activity in schizophrenia: a meta-analysis and a largesample study. Biomed Res Int 2015: 204628.

Yu Q, Sui J, Liu J, Plis SM, Kiehl KA, Pearlson G et al (2013). Disrupted correlation between low frequency power and connectivity strength of resting state brain networks in schizophrenia. Schizophr Res 143: 165-171.

Yu R, Chien YL, Wang HL, Liu CM, Liu CC, Hwang TJ et al (2014). Frequency-specific alternations in the amplitude of low-frequency fluctuations in schizophrenia. Hum Brain Mapp 35: 627-637.

Zalesky A, Fornito A, Egan GF, Pantelis C, Bullmore ET (2012). The relationship between regional and inter-regional functional connectivity deficits in schizophrenia. Hum Brain Mapp 33: 2535-2549.

Zang YF, He Y, Zhu CZ, Cao QJ, Sui MQ, Liang M et al (2007). Altered baseline brain activity in children with ADHD revealed by resting-state functional MRI. Brain Dev 29: 83-91.

Zou QH, Zhu CZ, Yang Y, Zuo XN, Long XY, Cao QJ et al (2008). An improved approach to detection of amplitude of low-frequency fluctuation (ALFF) for resting-state fMRI: fractional ALFF. J Neurosci Methods 172: 137-141.

Supplementary Information accompanies the paper on the Neuropsychopharmacology website (http://www.nature.com/npp) 